See Article page 80.

\section{Commentary: Handling mitral annulus calcification from behind the robotic console: The Pugachev's Cobra in cardiac surgery}

\author{
Gianluca Torregrossa, MD, ${ }^{\mathrm{a}}$ \\ Umberto Benedetto, MD, ${ }^{\mathrm{b}}$ and \\ Husam H. Balkhy, MD ${ }^{a}$
}

In aerobatics, the Pugachev's Cobra is a dramatic and demanding maneuver in which an airplane flying at a moderate speed suddenly raises its nose momentarily to the vertical position and slightly beyond before dropping it back down to normal. ${ }^{1}$ It is an impressive maneuver that demonstrates an aircraft's pitch control authority, high angle of attack stability and engine versus inlet compatibility, and the pilot's supreme skill. It was first performed in November 1989 by a Russian hero and experimental pilot, Viktor G. Pugachev, ${ }^{2}$ during the first appearance of the new jet fighter Sukhoi-27 to the Western World at the Paris Airshow. The Cobra defined a new standard for any pilot in terms of super-maneuverability and skills. The Pugachev's Cobra is not just an "airshow trick." It offers absolute superiority to the pilot capable of performing it during air-combat by suddenly inverting the prey-hunter position of one airplane pursued by another one (Figure 1).

In the current issue of the Journal, Loulmet and colleagues ${ }^{3}$ present their experience with 64 complex mitral valve robotic surgeries while dealing with mitral annulus calcification (MAC). These patients represent $12.8 \%$ of

\footnotetext{
From the ${ }^{\text {a Section }}$ of Cardiac and Thoracic Surgery, Department of Surgery, Robotic and Minimally Invasive Cardiac Surgery, The University of Chicago Medicine \& Biological Sciences, Chicago, Ill; and ${ }^{\mathrm{b}}$ Department of Cardiac Surgery, University of Bristol, Bristol Heart Institute, Bristol, United Kingdom.

Disclosures: Dr Balkhy receives proctoring fees from Intuitive Surgical. All other authors have nothing to disclose with regard to commercial support.

Received for publication Jan 5, 2020; revisions received Jan 5, 2020; accepted for publication Jan 5, 2020; available ahead of print Jan 31, 2020.

Address for reprints: Gianluca Torregrossa, MD, Section of Thoracic and Cardiac Surgery, Robotic and Minimally Invasive Cardiac Surgery, The University of Chicago Medicine \& Biological Sciences, 5841 S Maryland Ave, RM E-501, MC 5040, Chicago, IL 60637 (E-mail: gianluca.torregrossa@gmail.com).

J Thorac Cardiovasc Surg 2021;161:91-2

0022-5223/\$0.00

Published by Elsevier Inc. on behalf of The American Association for Thoracic Surgery

https://doi.org/10.1016/j.jtcvs.2020.01.025
}

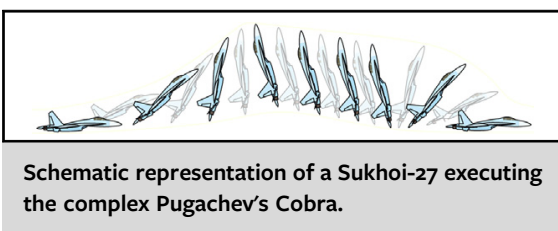

CENTRAL MESSAGE

Complex mitral valve repair is

enhanced using a robotic

approach with a dedicated,

experienced 2-surgeon team

even in the presence of complex

pathology and severe MAC.

the entire 500 robotic mitral case experiences operated on by the same surgical team during a 6-year period. ${ }^{4}$ Advanced robotic experience and a dedicated team approach were essential to guarantee a successful (mild to no mitral regurgitation) repair rate in $97 \%$ of patients by using a combination of advanced mitral valve repair techniques including MAC excision in $94 \%$ of cases, extensive atrial valve patch reconstruction in $30 \%$, sliding or hemi-sliding plasty of the posterior leaflet in $68 \%$, and papillary muscle repositioning in $10 \%$ of patients. Mortality at 30 days occurred in 2 patients.

These results were achieved because of the close collaboration between 2 experienced surgeons working together on every robotic mitral procedure. Of note, it is accepted as a routine that every commercial flight in the world is completed by 2 fully trained pilots, each capable of potentially flying solo. Aviation and medicine share the same standard of extreme safety and perfection, so it should come as no surprise to see 2 surgeons collaborate in everyday clinical practice when treating complex patients using such an innovative technique. Lack of appropriate coding and reimbursement and the increased demand on surgeon productivity have spread in medicine the misconception that a single pilot can do the same job of 2 in terms of safety and quality.

In this article, Loulmet and colleagues ${ }^{3}$ not only present their excellent results but also shed a new "light" (or in this case a new 3-dimensional stereoscopic view) on MAC pathology. The detailed description of the most frequent anatomic variants encountered during these complex surgeries and the clarity of the animations depicting one of the most complex procedures in cardiac surgery are refreshing. The authors emphasize the fact that they are an experienced mitral surgical team, but they also discuss 


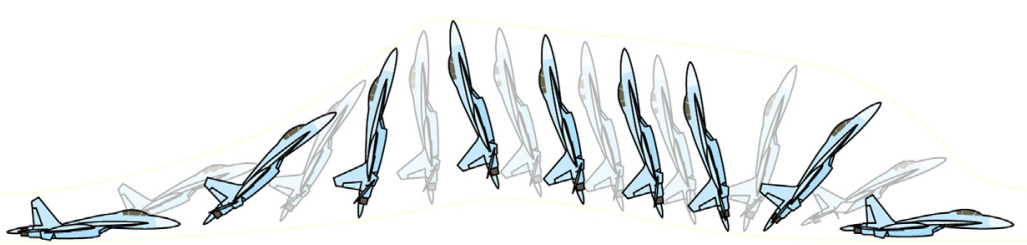

FIGURE 1. Schematic representation of a Sukhoi-27 executing the complex Pugachev's Cobra. The picture copyright belongs to Henry W., User:Henrickson (https://en.wikipedia.org/wiki/User:Henrickson). The picture is available at https://en.wikipedia.org/wiki/User:Henrickson\#/media/File:Su-27_Cobra_ 2b.png, with CC licensing BY-SA 3.0 with full free authorization to copy and redistribute the material in any medium or format after providing appropriate credit. (https://creativecommons.org/licenses/by-sa/3.0/).

how the robotic view has enhanced their ability to properly analyze the mitral valve leaflets and subvalvular apparatus. The robotic dynamic retractor offers superior exposure of each segment of the mitral valve, allowing accurate placement of the critical left ventricular sutures to secure the posterior patch and increasing the probability of achieving a successful mitral valve repair even in these complex cases.

For several years, the Pugachev's Cobra remained the dream and nightmare of every Western jet fighter pilot. Western countries were unable to replicate the maneuver, so they started to develop a new generation of planes with advanced vectoring technology allowing them to perform the Cobra. Meanwhile, during the 1990s, Western jet pilots adopted an intense training enabling them to adopt the $\mathrm{Pu}$ gachev's maneuver as part of their armamentarium as aircombat fighters. Training, dedication, and technology were the mix necessary to demystify the Cobra and bring it into the hands of every well-trained fighter pilot around the world.

In the current era of transcatheter technology, there has been a tendency to accept suboptimal results as part of our surgical success, including the increased need for pacemaker implantation, perivalvular leaks, valve thrombosis, and less than perfect repair of the mitral valve. This article by Loulmet and colleagues ${ }^{3}$ sets a new benchmark of what a minimally invasive mitral valve center of excellence can offer to patients, even to those with more complex disease.

The aim to reproduce perfection in a repetitive matter, as presented by Loulmet and colleagues, ${ }^{3}$ without compromising the medium- and long-term results, should be the focus of the younger generation of cardiac surgeons. Intense training and industry support toward robotic and minimally invasive cardiac surgery are essential to reproduce these results and to guarantee the Pugachev's Cobra becomes part of the everyday practice offered to our patients.

\section{References}

1. Wikipedia.org. Cobra Maneuver. Available at: https://en.wikipedia.org/wiki/ Cobra_maneuver. Accessed February 19, 2020.

2. Wikipedia.org. Viktor Pugachev. Available at: https://en.wikipedia.org/wiki/ Viktor_Pugachev. Accessed February 19, 2020.

3. Loulmet DF, Ranganath NK, Neragi-Miandoab S, Koeckert MS, Galloway AC, Grossi EA. Advanced experience allows robotic mitral valve repair in the presence of extensive mitral annular calcification. J Thorac Cardiovasc Surg. 2021;161:80-8.

4. Loulmet DF, Ranganath NK, Neuburger PJ, Nampiaparampil RG, Galloway AC, Grossi EA. Can complex mitral valve repair be performed with robotics? An institution's experience utilizing a dedicated team approach in 500 patients. Eur J Cardiothorac Surg. 2019;56:470-8. 